\title{
Platinum Polyoxoniobates have a potential as an anticancer agent
}

\author{
Anna V. Yudkina \\ ICBFM SB RAS, Novosibirsk, Russia \\ NSU, Novosibirsk, Russia \\ yudkinaanya@gmail.com \\ Pavel A. Abramov \\ NIIC SB RAS, Novosibirsk, Russia \\ abramov@niic.nsc.ru
}

\author{
Ivan P. Vokhtantsev \\ ICBFM SB RAS, Novosibirsk, Russia \\ NSU, Novosibirsk, Russia \\ ivanvohtancev@gmail.com \\ Inga R. Grin \\ ICBFM SB RAS, Novosibirsk, Russia \\ NSU, Novosibirsk, Russia \\ grin@niboch.nsc.ru
}

\author{
Maxim N. Sokolov \\ NIIC SB RAS, Novosibirsk, Russia \\ caesar@niic.nsc.ru \\ Dmitry O. Zharkov \\ ICBFM SB RAS, Novosibirsk, Russia \\ NSU, Novosibirsk, Russia \\ dzharkov@niboch.nsc.ru
}

\begin{abstract}
It is well-known that platinum coordination complexes are widely used anticancer agents. Here we have investigated the biological properties of platinum polyoxometalates - inorganic coordination compounds with a $\mathbf{P t}$, as a potential antitumor agent.
\end{abstract}

damage

Keywords - polyoxometalates, DNA polymerases, DNA

\section{Motivation and Aim}

\section{Motivation}

Platinum complexes are among the most commonly prescribed drugs in cancer therapy. However, they have serious restrictions such as significant toxicity to healthy tissues and cell resistance. One strategy to overcome these limitations is the development of new, improved platinum drugs. One of such promising group of compounds could be platinum polyoxometalates - platinum complexes liganded with cluster anions consisting of oxygen atoms and transition metals. Their properties related to a potential use as anticancer drugs have never been studied.

Aim

In this work, we aim to investigate the cytotoxic effect of platinum (IV) polyoxoniobate of the $\left[\mathrm{Nb}_{6} \mathrm{O}_{19}\left\{\mathrm{Pt}(\mathrm{OH})_{2}\right\}\right]_{2}$ structure containing two platinum centers and two polynuclear Lindqvist type anions [1].

\section{Methods}

First, we have tested the effect of platinum (IV) polyoxoniobate on the activity of a number of DNA polymerases belonging to different families (Klenow fragment of Escherichia coli DNA polymerase I, bacteriophage RB69 DNA polymerase, human DNA polymerases $\beta$ and $\kappa$, DNA polymerase IV from Sulfolobus solfataricus) to investigate in vitro possible mechanisms of its cytotoxicity. Moreover, the cytotoxic effect of platinum (IV) polyoxoniobate was investigated in several cell lines. The nature of platinum (IV) polyoxoniobate adduct with DNA was confirmed by MALDI-mass spectrometry.

\section{Results}

It has been shown that platinum (IV) polyoxoniobate of the structure $\left[\mathrm{Nb}_{6} \mathrm{O}_{19}\left\{\mathrm{Pt}(\mathrm{OH})_{2}\right\}\right]_{2}$ demonstrates properties similar to that of cisplatin: it was capable of forming covalent bulky adducts with DNA, which interfere with the primer elongation by the studied DNA-polymerases, and mostly contribute to studied compound cytotoxicity. At the same time, platinum (IV) polyoxoniobate did not act as an inhibitor of DNA polymerases.

\section{ACKNOWLEDGMENT}

The authors thank Facilities of the Joint Center for Genomic, Proteomic and Metabolomics Studies Institute of Chemical Biology and Fundamental Medicine, Novosibirsk, Russia.

This study was supported by the Russian Foundation for Basic Research (19-44-543011-r-mol-a) and Novosibirsk region.

\section{REFERENCES}

[1] Abramov P. A. et al. (2015) Platinum polyoxoniobates. Chem. Commun. 51(19): 4021-4023 